\title{
MONITORAMENTO DA QUALIDADE DA ÁGUA TRATADA POR SISTEMAS DE DESFLUORETAÇÃO DE ÁGUAS SUBTERRÂNEAS
}

\author{
Gisele Steil Rodrigues ${ }^{1}$ \\ Eduardo A. Lobo ${ }^{2}$ \\ Alcido Kirst ${ }^{3}$ \\ Adilson Ben da Costa
}

\section{RESUMO}

O principal objetivo deste estudo foi avaliar a eficiência de sistemas de desfluoretação de águas para abastecimento público, com o propósito de contribuir para a redução da ocorrência de fluorose dental. Assim, foram construídos e instalados cinco filtros, com seis quilogramas de carvão ativado de osso, os quais foram monitorados durante dez meses. Os resultados indicaram que os filtros se mantiveram eficientes durante sete meses, tratando $4.715 \pm 881$ litros de água $(\mathrm{n}=5)$, mantendo a concentração de íons fluoretos em níveis inferiores a 1,5 $\mathrm{mgL}^{-1}$ conforme do especificado pela Portaria ${ }^{\circ}$ 2914, Ministério da Saúde (a concentração original da água bruta era da ordem de $3,2 \mathrm{mgL}^{-1}$. Estes resultados demostram, assim, o potencial deste sistema para atender as necessidades de abastecimento coletivo, como em escolas, universidades e estabelecimentos comerciais, por exemplo.

Palavras-chave: Íons fluoreto. Monitoramento. Filtros de desfluoretação. Carvão ativado de osso.

\begin{abstract}
The main goal of study was to evaluate the efficiency of waters desfluoretation systems of for public supply, the purpose of contribute to reducing of the occurrence of dental fluorosis. Thus, five filters were constructed and installed using six kilograms of activated bone charcoal, which were monitoring for ten months. The results indicated that the filters remained efficient for seven months, treating 4,715 \pm 881 liters of water $(n=5)$, maintaining the concentration of fluoride ions at levels below $1.5 \mathrm{mgL}^{-1}$ according to of the specified by Ordinance $\mathrm{n}^{\circ}$. 2914, Ministry of health (the original concentration of the raw water was approximately $3.2 \mathrm{mg} \mathrm{L}^{-1}$. These results demonstrate thus the potential of this system to serve the collective supply necessities like in schools, universities and commercial establishments, for example.
\end{abstract}

Keywords: Fluoride. Monitoring. Desfluoretation filters. Activated bone charcoal.

\footnotetext{
${ }^{1}$ Acadêmica do Curso de Química Industrial da Universidade de Santa Cruz do Sul, RS. <steilgisele@gmail.com>

2 Professor do Departamento de Biologia e Farmácia da Universidade de Santa Cruz do Sul, RS. <lobo@unisc.br>

${ }_{3}^{3}$ Professor do Departamento de Química e Física da Universidade de Santa Cruz do Sul, RS. < $\underline{\text { alcido@unisc.br> }}$

${ }^{4}$ Professor do Departamento de Biologia e Farmácia da Universidade de Santa Cruz do Sul, RS. <
} 


\section{INTRODUÇÃO}

O sistema público de abastecimento de água de muitos municípios brasileiros limitase apenas à área urbana e, em virtude disto, as comunidades rurais utilizam a água proveniente de poços e fontes naturais, denominados sistemas alternativos de abastecimento, os quais são, muitas vezes, administrados pelas próprias comunidades, na forma de cooperativas (COSTA et al., 2013). No entanto, em muitos casos, a água utilizada para abastecimento não apresenta condições de ser consumida sem um tratamento prévio, devido ao fato de algumas características físicas, químicas ou microbiológicas apresentarem valores em desacordo com os padrões recomendados para o consumo humano (COSTA et al., 2013).

De acordo com Soares et al. (2008), um dos fatores preocupantes tem sido a ocorrência de fluorose dental em crianças que vem sendo diagnosticada no Brasil, como uma patologia associada ao consumo prolongado de água com excesso de íons fluoreto (SOARES et al., 2008). Apesar de o flúor ser um importante fator no combate à cárie, o consumo de água com excesso de íons fluoreto pode resultar em sérios problemas ósseos, especialmente em crianças (COSTA, 2010).

A concentração de fluoreto é um parâmetro relevante para avaliação de qualidade nas águas de consumo, seja pela possibilidade de prevenção da cárie dentária, quando presente em níveis adequados, seja pelo potencial de provocar a fluorose dental, quando em níveis elevados. Estabelecer níveis de segurança para o fluoreto em águas de consumo é uma medida imprescindível de proteção à saúde bucal (FRAZÃO et al., 2011).

Segundo RIBEIRO 2011, a fluorose dental é uma anomalia ligada à estética do esmalte dentário, suas características vão desde finas linhas brancas cruzando os dentes em forma de traços até várias formas de erosão. Os efeitos dependem da concentração e tempo de exposição ao fluoreto, durante a infância no período de formação dos dentes permanentes.

Toda água destinada ao consumo humano deve obedecer aos padrões de qualidade estabelecidos na Portaria $\mathrm{n}^{\mathrm{o}}$ 2914, de 12 de dezembro de 2011, Ministério da Saúde, que permite um limite máximo de concentração de íons fluoreto em águas de abastecimento para consumo humano de $1,5 \mathrm{mg} \mathrm{L^{-1 }}$. Porém, a elevada concentração de flúor das águas subterrâneas das regiões dos Vales do Rio Pardo e Rio Taquari, RS, tem apresentado efeito direto sobre a saúde das populações abastecidas por estas fontes (SOARES et al., 2008).

O laboratório de Hidrologia da Universidade de Santa Cruz do Sul, RS, (UNISC) preocupado com o problema, vem desenvolvendo estudos para reduzir a concentração de íons fluoreto na água de abastecimento até valores adequados ao consumo humano. Estudos anteriores (COSTA et al., 2010) demonstraram a eficiência da utilização de carvão ativado de Revista Jovens Pesquisadores, Santa Cruz do Sul, v. 5, n. 3, p. 49-62, 2015. 
osso bovino, como meio adsorvente, em filtros domésticos de desfluoretação, utilizando amostras de água subterrânea com concentrações de íons fluoreto na ordem de $4,0 \mathrm{mg} \mathrm{L}^{-1}$.

Neste contexto, este estudo apresenta os mais recentes resultados obtidos no desenvolvimento de sistemas de desfluoretação de águas subterrâneas, o qual foi instalado no Campus de Santa Cruz do Sul da Universidade de Santa Cruz do Sul, Rio Grande do Sul.

\section{FUNDAMENTAÇÃO TEÓRICA}

Atualmente, são cada vez mais frequentes os relatos de problemas associados ao abastecimento de água para consumo humano, mesmo em regiões com alta disponibilidade hídrica. Observa-se que a combinação do crescimento excessivo de demandas localizadas e da degradação da qualidade da água tem como resultado o fato de que diversas cidades brasileiras enfrentam dificuldades em manter, de forma estável e com qualidade, o abastecimento de água em suas comunidades (COSTA, 2010).

Muito se tem discutido a importância da água para a manutenção da vida e das diversas atividades humanas, no que se refere a dois importantes aspectos: a mudança de paradigma quanto à gratuidade da água e a necessidade urgente de medidas que revertam o seu atual quadro de degradação, dado seu caráter finito (JÚNIOR et al., 2009).

As águas, tanto superficiais quanto subterrâneas, precisam de monitoramento adequado e, pelo fato de as condições das águas subterrâneas serem mais difíceis de observar, é imprescindível que recursos e atenção sejam dedicados ao monitoramento apropriado das fontes e da qualidade das águas subterrâneas (ANA, 2011 apud MORRIS et al., 2003).

Segundo a Organização das Nações Unidas - ONU (2006), 50\% da taxa de doenças e mortes nos países em desenvolvimento ocorrem por falta de água ou pela sua contaminação (EXTERCKOTER et al., 2008 apud ONU, 2006).

Fluorose dental é uma deficiência na mineralização do esmalte devido à ingestão diária de fluoreto durante o desenvolvimento dental. Como há uma relação linear dose-efeito, as consequências dependem da concentração de fluoreto e do tempo em que ela é mantida constante nos líquidos teciduais durante a mineralização do esmalte. Assim, a prevalência de fluorose dental em populações abastecidas por água fluoretada depende do tempo que determinada concentração é mantida constante durante o desenvolvimento dental. Dados epidemiológicos sobre essa questão ainda são carentes na literatura (CATANI et al., 2007).

A fluorose dental leve causa apenas alterações estéticas, caracterizadas por pigmentação branca do esmalte dentário. A fluorose dental moderada e severa, caracterizada Revista Jovens Pesquisadores, Santa Cruz do Sul, v. 5, n. 3, p. 49-62, 2015. 
por manchas amareladas ou marrons, além de defeitos estruturais no esmalte, apresenta repercussões estéticas, morfológicas e funcionais. A fluorose sistêmica, gerada por ingestão de altas concentrações de flúor (acima de $8,0 \mathrm{mg} \mathrm{L}^{-1}$ ), provoca alterações esqueléticas, articulares, neurológicas e nefrológicas, dentre outras (MOYSÉS et al., 2002).

A fluorose esquelética afeta tanto crianças quanto adultos. Os sintomas iniciais incluem formigamento nos membros, fraqueza muscular, fadiga crônica, depósito de cálcio nos ossos e ligamentos anormais. No estágio avançado, ocorre osteoporose. Em casos mais raros, pode ocorrer câncer ósseo (MEENAKSHI e MAHESHWRI, 2006; OMS, 2005). Diferentes alternativas têm sito propostas na literatura para a remoção parcial ou completa de íons fluoreto da água, no entanto, o desenvolvimento de filtros de adsorção com carvão ativado de osso tem merecido destaque nos últimos anos (COSTA et al. 2013).

O carvão de osso é um material carbonoso onde se distribui uma estrutura porosa de hidroxiapatita $\left(\mathrm{Ca}_{10}\left(\mathrm{PO}_{4}\right)_{6}(\mathrm{OH})_{2}\right)$, sendo conhecido também por outras denominações como carvão de osso, carvão negro ou pigmento preto. Aproximadamente 70 a $75 \%$ do carvão de osso é hidroxiapatita, o principal constituinte inorgânico dos ossos de animais, além de pequenas quantidades de calcita $\left(\mathrm{CaCO}_{3}\right)$ devido à presença de $\mathrm{CO}_{2}$ no forno durante o processo de produção (RIBEIRO, 2011). 


\section{MATERIAIS E MÉTODOS}

Para a avaliação da eficiência do sistema de desfluoretação, foram construídos e instalados, ao todo, cinco filtros de desfluoretação em fibra de vidro (PolyGlass®), disponibilizado pela empresa Pentair Hidro Filtros do Brasil Indústria e Comércio de Filtros Ltda. (Caxias do Sul, RS), contendo em seu interior $6 \mathrm{~kg}$ de carvão ativado de osso bovino (20-60 mesh), disponibilizado pela indústria Bonechar - Carvão Ativado do Brasil Ltda. (Maringá, PR). Na saída de cada filtro foi conectado um filtro auxiliar, de fibra de polietileno, com a finalidade de auxiliar na remoção de turbidez (Figura 1).

Figura 1 - Diagrama de um dos sistemas de filtração desenvolvido neste estudo

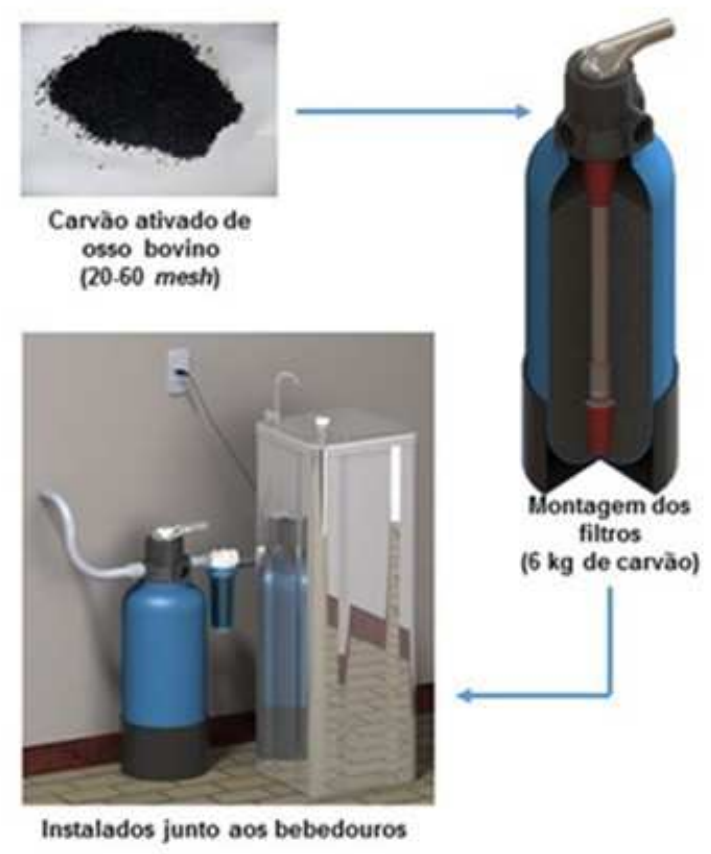

Os filtros foram instalados nos blocos 8 e 12 do campus de Santa Cruz do Sul, da Universidade de Santa Cruz do Sul, o qual é abastecido por água subterrânea proveniente de dois poços subterrâneos, cujas concentrações de íons fluoreto variam entre 2,5 e 4,0 mgL $\mathrm{mL}^{-1}$ (Figura 2). 
Figura 2 - Imagens dos filtros testes F1, F2, F3, F4 e F5 instalados em pontos de consumo no campus da UNISC.
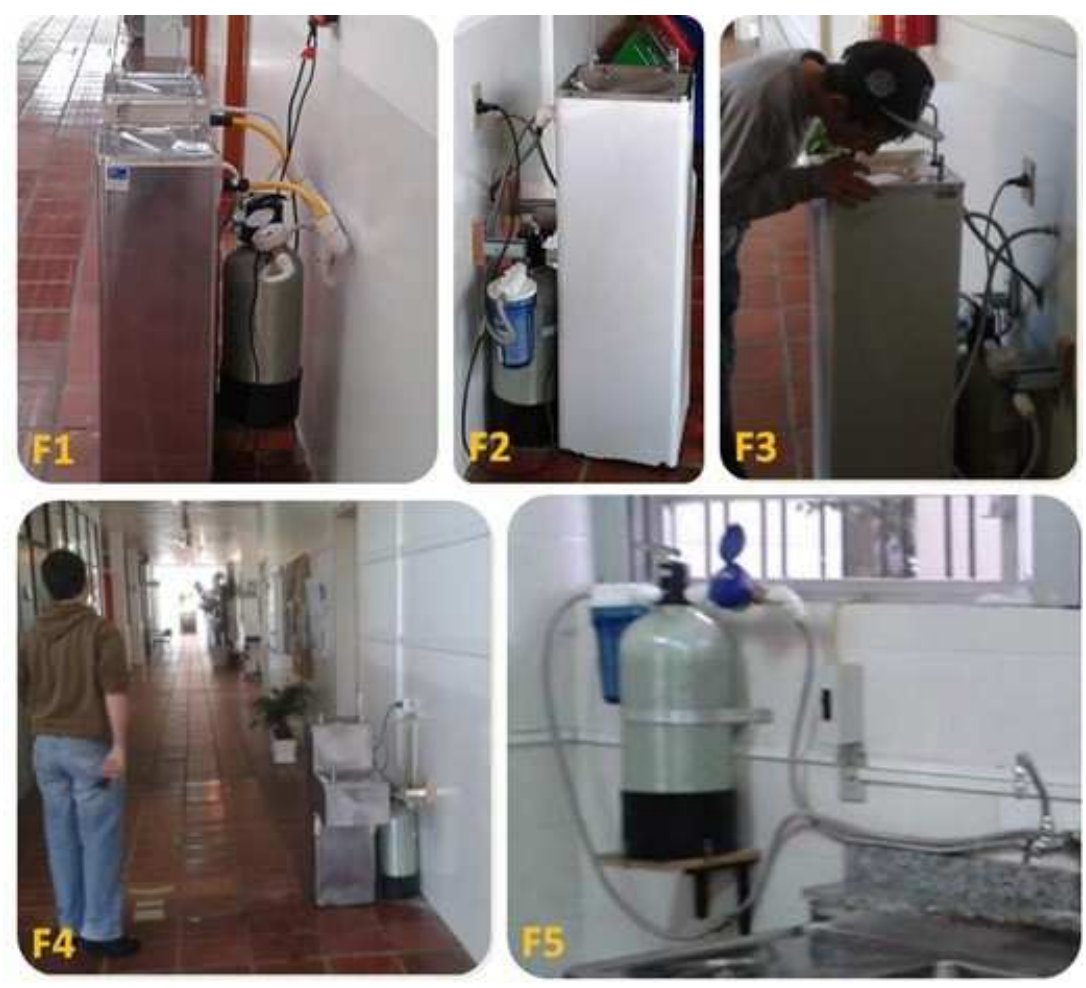

Durante os meses de agosto de 2013 a junho de 2014, a qualidade da água bruta e tratada por cada um dos sistemas de desfluoretação foi monitorada. Sendo determinados os seguintes parâmetros: condutividade elétrica, flúor, $\mathrm{pH}$, turbidez e mensalmente os seguintes parâmetros: alcalinidade de bicarbonatos, alcalinidade de carbonatos, alcalinidade de hidróxidos, cálcio, cloretos, dureza total, ferro total, fósforo total, magnésio, manganês, potássio, sódio, sólidos totais dissolvidos e sulfatos. As metodologias analíticas utilizadas para a determinação das variáveis físicas, químicas e biológicas, encontram-se descritas em APHA (2005).

A vida útil do sistema é definida no momento em que os resultados da água tratada atingirem valores superiores a $1,5 \mathrm{mg} \mathrm{L}^{-1}$ íons fluoreto, limite máximo estabelecido pela Portaria no 2914, de 12 de dezembro de 2011, do Ministério da Saúde, que indica a saturação da fase estacionária. Os primeiros 50 litros de água tratada foram considerados como purga do sistema e descartados, para eliminar a turbidez, originária do carvão. 


\section{RESULTADOS E DISCUSSÕES}

O monitoramento da qualidade da água subterrânea bruta, proveniente de dois poços utilizados como fonte de abastecimento, foi de extrema importância para a avaliação dos parâmetros físicos, químicos e biológicos originais da água, podendo assim determinar as alterações provenientes do sistema de filtração, que foram avaliados neste estudo.

Os resultados obtidos da água bruta e da água tratada indicam uma água alcalina, bicabornatada, sódica, rica em íons bicarbonatos e sódio.

As Figuras 3 e 4 apresentam os resultados de alcalinidade, bicarbonatos e sódio.

Figura 3 - Valores médios para alcalinidade e bicarbonatos, obtidos no monitoramento da água bruta 1 e 2 (água subterrânea natural, $n=8)$ e na água tratada pelos filtros $1(n=8)$, filtro $2(n=7)$, filtro $3(n=8)$, filtro $4(n=8)$, filtro $5(n=7)$ e respectivos desvios-padrão.

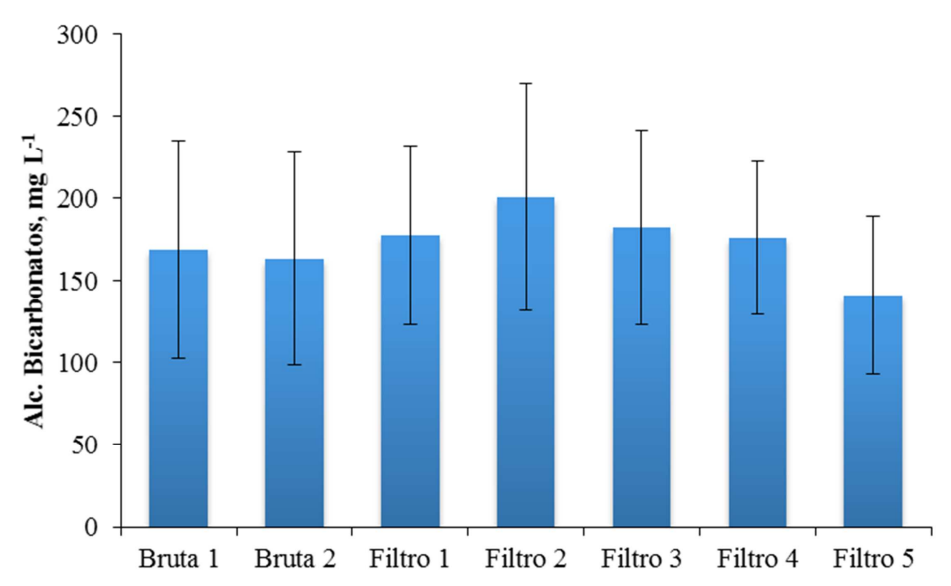

Figura 4 - Valores médios para sódio, obtidos no monitoramento da água bruta 1 e 2 (água subterrânea natural, $n=10)$ e na água tratada pelos filtros $1(n=10)$, filtro $2(n=9)$, filtro $3(n=10)$, filtro $4(n=10)$, filtro $5(n=8)$ e respectivos desvios-padrão. A linha pontilhada representa o valor máximo permitido (VMP) pela portaria $n^{\circ}$ 2914, do Ministério da Saúde, Brasil.

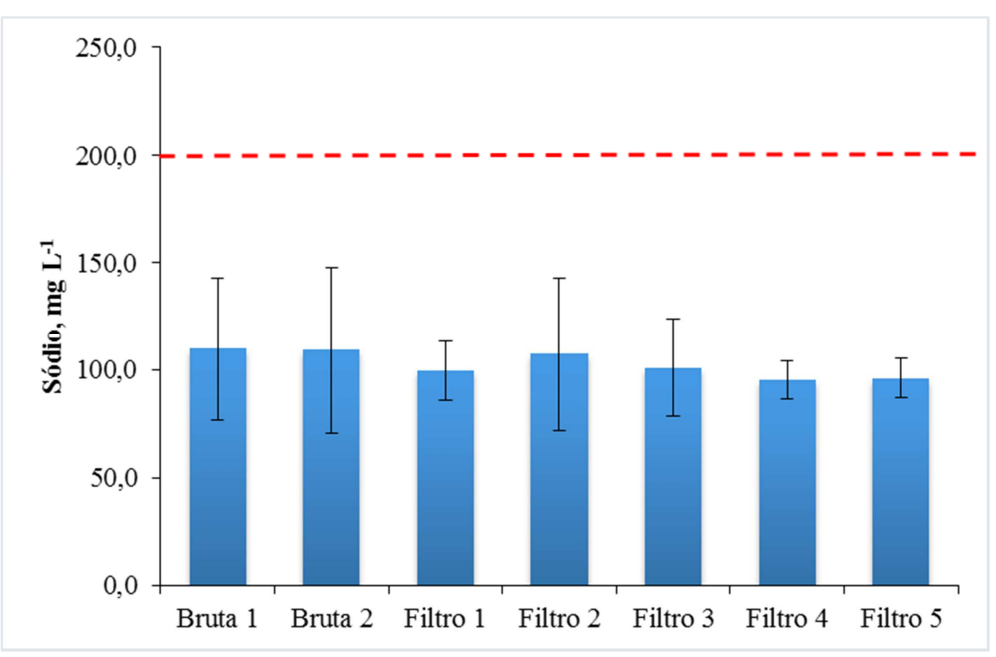

Revista Jovens Pesquisadores, Santa Cruz do Sul, v. 5, n. 3, p. 49-62, 2015. 
A Tabela 1 apresenta os resultados referentes ao período de monitoramento da água bruta. Cabe destacar que entre todos os parâmetros avaliados, apenas a concentração de íons fluoreto apresenta-se em desacordo com os padrões estabelecidos para consumo humano, segundo a Portaria n 2914 (BRASIL, 2011).

Tabela 1 - Resultados obtidos no monitoramento da água subterrânea bruta, utilizada como fonte de abastecimento para os sistemas de desfluoretação.

\begin{tabular}{|c|c|c|c|}
\hline Parâmetros & VMP & Poço 1 & Poço 2 \\
\hline Alcalinidade bicarbonatos, $\mathrm{mg} \mathrm{L}^{-1}\left(\mathrm{CaCO}_{3}\right)$ & $*$ & $169 \pm 6 n=8$ & $164 \pm 8 n=8$ \\
\hline Alcalinidade carbonatos, $\mathrm{mg} \mathrm{L}^{-1}\left(\mathrm{CaCO}_{3}\right)$ & * & $53 \pm 12 n=8$ & $62 \pm 17 n=8$ \\
\hline Alcalinidade hidróxidos, $\mathrm{mg} \mathrm{L}^{-1}\left(\mathrm{CaCO}_{3}\right)$ & $*$ & $0 \mathrm{n}=8$ & $0 \mathrm{n}=8$ \\
\hline Cálcio, $\mathrm{mg} \mathrm{L}^{-1}$ & $*$ & $0,79 \pm 0,34 \mathrm{n}=10$ & $0,80 \pm 0,26 \mathrm{n}=10$ \\
\hline Cloretos, $\mathrm{mg} \mathrm{L}^{-1}\left(\mathrm{Cl}^{-1}\right)$ & 250 & $25,6 \pm 22,2 \mathrm{n}=8$ & $21,9 \pm 6,2 \mathrm{n}=8$ \\
\hline Condutividade elétrica, $\mu \mathrm{S} \mathrm{cm}^{-1}$ & * & $503 \pm 53 n=47$ & $518 \pm 37 n=41$ \\
\hline Dureza total, $\mathrm{mg} \mathrm{L}^{-1}\left(\mathrm{CaCO}_{3}\right)$ & 500 & $2,2 \pm 1,0 \mathrm{n}=10$ & $2,2 \pm 1,3 \mathrm{n}=10$ \\
\hline Ferro, $\mathrm{mg} \mathrm{L}^{-1}$ & 0,3 & $<0,2 \mathrm{n}=10$ & $<0,2 \mathrm{n}=10$ \\
\hline Fluoretos, $\mathrm{mg} \mathrm{L}^{-1}$ & 1,5 & $3,2 \pm 0,4 \mathrm{n}=48$ & $3,2 \pm 0,4 n=42$ \\
\hline Fósforo total, $\mathrm{mg} \mathrm{L}^{-1}(\mathrm{P})$ & $*$ & $0,04 \pm 0,10 \mathrm{n}=10$ & $0,01 \pm 0,01 \mathrm{n}=10$ \\
\hline Magnésio, $\mathrm{mg} \mathrm{L}^{-1}$ & $*$ & $<0,03 n=10$ & $<0,1 \mathrm{n}=10$ \\
\hline Manganês, mg L $\mathrm{L}^{-1}$ & * & $<0,04 n=10$ & $<0,03 n=10$ \\
\hline $\mathrm{pH}$ & 6,0 a 9,5 & $9,0 \pm 0,2 \mathrm{n}=47$ & $9,0 \pm 0,2 \mathrm{n}=41$ \\
\hline Potássio, mg L ${ }^{-1}$ & $*$ & $<0,3 n=10$ & $<0,3 n=10$ \\
\hline Sódio, $\mathrm{mg} \mathrm{L}^{-1}$ & 200 & $100,9 \pm 14,6 n=10$ & $109,3 \pm 38,6 n=10$ \\
\hline Sólidos totais dissolvidos, $\mathrm{mg} \mathrm{L}^{-1}$ & 1.000 & $364,5 \pm 38,6 n=47$ & $375,2 \pm 27,0 n=41$ \\
\hline Sulfato, $\mathrm{mg} \mathrm{L}^{-1}\left(\mathrm{SO}_{4}^{-2}\right)$ & 250 & $25,9 \mathrm{n}=1$ & $26,7 n=1$ \\
\hline Turbidez, uT & 5 & $<0,3 \mathrm{n}=47$ & $<0,2 \mathrm{n}=41$ \\
\hline
\end{tabular}

Onde: (VMP) Valor máximo permitido pela Portaria $\mathrm{n}^{\circ}$ 2914, do Ministério da Saúde; (*) parâmetros não citados pela Portaria no 2914, do Ministério da Saúde.

O monitoramento da água tratada permitiu identificar um aumento na concentração de fósforo que pode ter ocorrido pela variação de vazão ou no volume correspondente a cada coleta (Figura 5). 
Figura 5 - Valores médios para fósforo, obtidos no monitoramento da água bruta 1 e 2 (água subterrânea natural, $n=10)$ e na água tratada pelos filtros $1(n=10)$, filtro $2(n=9)$, filtro $3(n=9)$, filtro $4(n=10)$, filtro $5(n=8)$ e respectivos desvios-padrão.

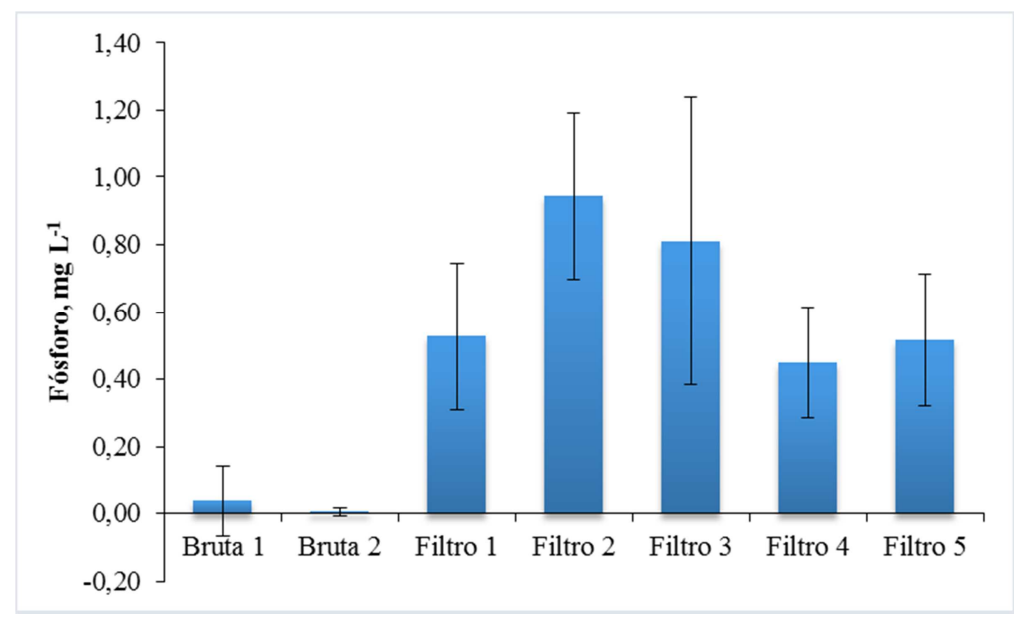

As Figuras 6,7 e 8 apresentam os resultados de pH, sólidos totais dissolvidos e turbidez, os mesmos mantiveram-se dentro dos parâmetros estabelecidos pela Portaria $\mathrm{n}^{\circ}$ 2914, do Ministério da Saúde (BRASIL, 2011).

Figura 6 - Resultados do monitoramento do $\mathrm{pH}$ da água bruta e da água tratada dos filtros F1, F2, F3, F4 e F5. As linhas pontilhadas limitam a faixa de $\mathrm{pH}$ determinada na portaria nº 2914, do Ministério da Saúde, Brasil.

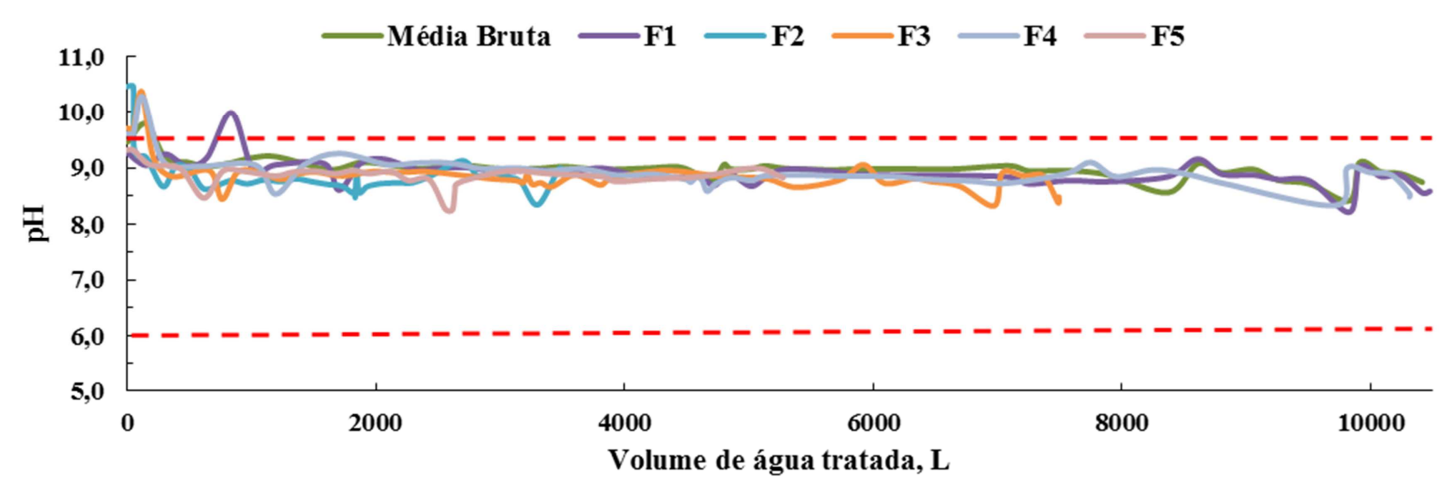

Como pode ser observado nas primeiras coletas de amostra, ocorreu uma elevação do $\mathrm{pH}$, que se deu como um agravante por apresentar uma água bruta muito alcalina. 
Figura 7 - Resultados do monitoramento de sólidos totais dissolvidos da água bruta e da água tratada dos filtros F1, F2, F3, F4 e F5. A linha pontilhada representa o valor máximo permitido (VMP) pela portaria $\mathrm{n}^{\circ}$ 2914, do Ministério da Saúde, Brasil.

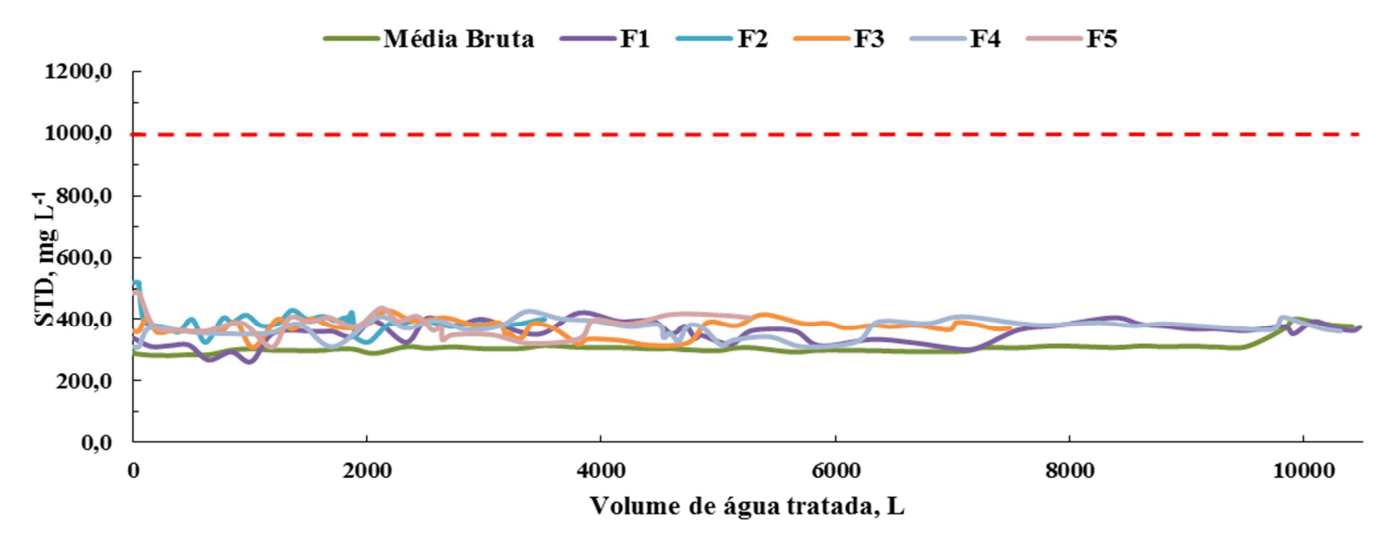

Figura 8 - Resultados do monitoramento de turbidez da água bruta e da água tratada pelos filtros F1, F2, F3, F4 e F5. A linha pontilhada representa o valor máximo permitido (VMP) pela portaria $\mathrm{n}^{\circ}$ 2914, do Ministério da Saúde, Brasil.

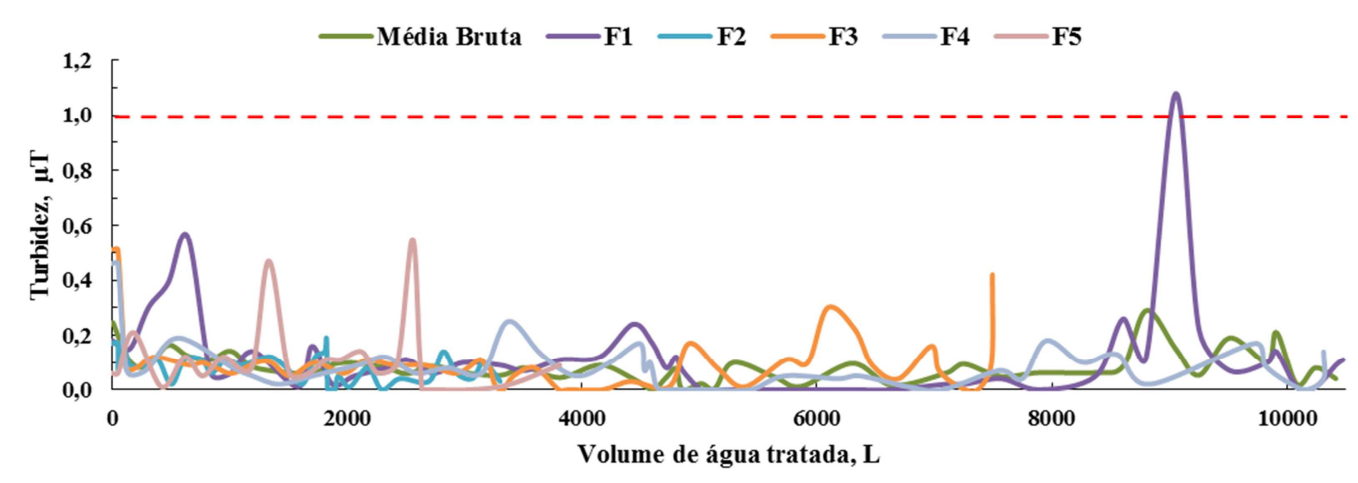

Os resultados alcançados dos filtros F1, F3, F4 e F5 mostram a eficiência do sistema para a remoção parcial de íons fluoreto. O período de eficiência do sistema foi de sete meses (198,3 $\pm 14,1$ dias), sendo assim tratados em média $4.715 \pm 881$ litros de água para consumo humano, e uma média de 25,0 \pm 4,1 de volume de água por dia, os parâmetros monitorados mantiveram-se dentro do especificado pela Portaria $n^{\circ} 2914$, Ministério da Saúde. A alta variação nos resultados apresentados pelos filtros (coeficiente de variação de 18,7\%) deve-se as diferentes condições de vazão em que foram operados, visto que atuaram em condições reais de uso. O F2 continua em operação, pois sua fase estacionária não saturou até o momento de fechamento deste artigo.

A Figura 9 apresenta os resultados do monitoramento da concentração dos íons fluoreto referente aos cinco filtros de desfluoretação avaliados neste estudo. 
Figura 9 - Resultados do monitoramento da concentração de íons fluoreto da água bruta e da água tratada pelos filtros F1, F2, F3, F4 e F5. A linha pontilhada representa o valor máximo permitido (VMP) pela portaria $\mathrm{n}^{\circ} 2914$, do Ministério da Saúde, Brasil.

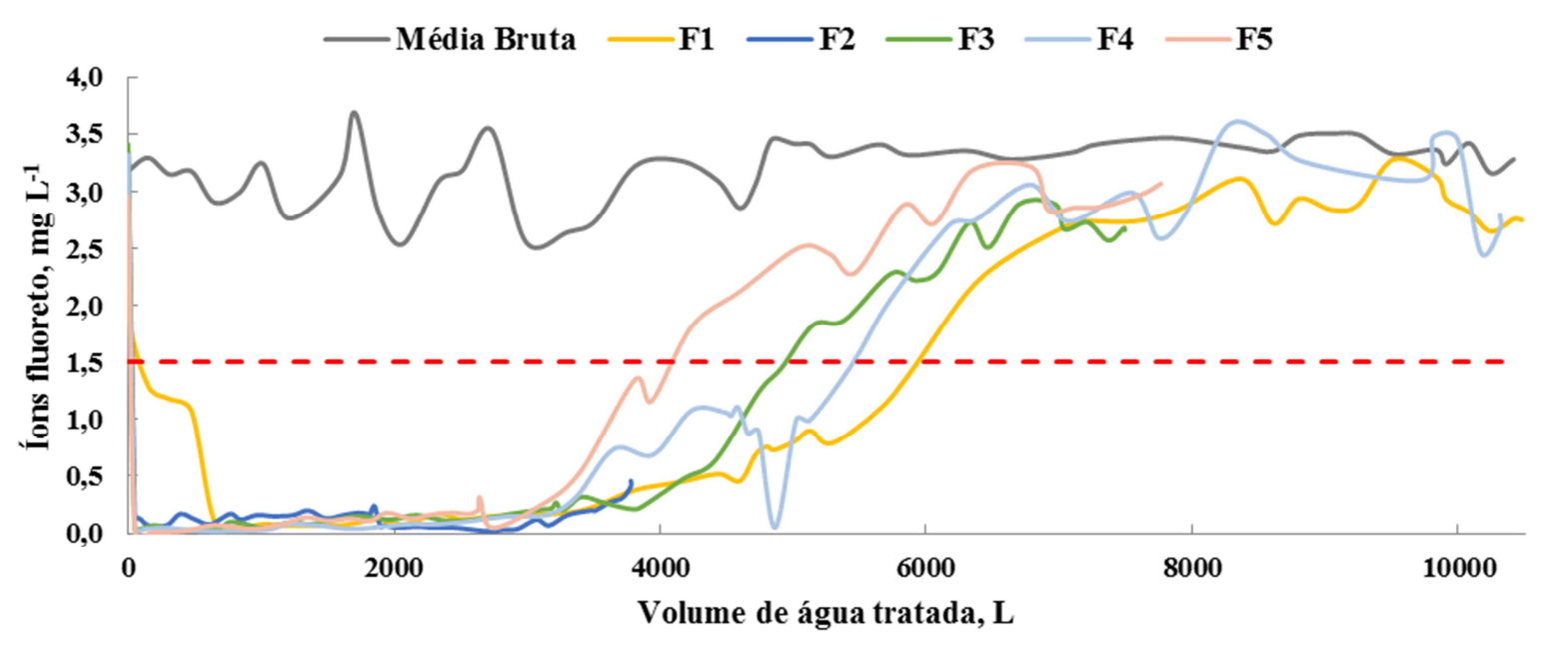

Os resultados médios e desvios-padrão do monitoramento da concentração de íons fluoretos e o volume de água tratada pelos filtros de desfluoretação foram para o filtro 1, 1,39 $\pm 1,2(\mathrm{n}=48)$, tratando 5640,9 L; filtro 2 em operação; filtro 3, 0,96 $\pm 1,1(\mathrm{n}=44), 4910,9 \mathrm{~L}$; filtro 4, 1,44 $\pm 1,3(\mathrm{n}=44), 5435,9 \mathrm{~L}$ e filtro 5, 1,14 $\pm 1,3(\mathrm{n}=38), 3811,5 \mathrm{~L}$; cabe salientar que o filtro 5 obteve um volume de água tratada menor, devido a sua vazão ter sido maior, sendo assim, saturando o carvão ativado de osso rapidamente.

A Tabela 2 apresenta os resultados dos parâmetros monitorados da água tratada, referente ao período de monitoramento, sendo que os resultados apresentam-se dentro dos valores estabelecidos pela Portaria no 2914, do Ministério da Saúde (BRASIL, 2011), destacando os resultados de íons fluoreto. 


\begin{tabular}{|c|c|c|c|c|c|c|}
\hline Parâmetros & VMP & Filtro 1 & Filtro 2 & Filtro 3 & Filtro 4 & Filtro 5 \\
\hline Alcalinidade bicarbonatos, $\mathrm{mg} \mathrm{L}^{-1}\left(\mathrm{CaCO}_{3}\right)$ & $*$ & $177,5 \pm 54,2 n=8$ & $201,1 \pm 69,2 \mathrm{n}=7$ & $182,3 \pm 59,2 \mathrm{n}=8$ & $176,3 \pm 46,6 n=8$ & $141,4 \pm 48,1 \mathrm{n}=7$ \\
\hline Alcalinidade carbonatos, $\mathrm{mg} \mathrm{L}^{-1}\left(\mathrm{CaCO}_{3}\right)$ & $*$ & $42,5 \pm 10,7 \mathrm{n}=8$ & $38,9 \pm 11,9 \mathrm{n}=7$ & $44,5 \pm 16,8 \mathrm{n}=8$ & $43,0 \pm 7,3 \mathrm{n}=8$ & $47,4 \pm 12,3 \mathrm{n}=7$ \\
\hline Alcalinidade hidróxidos, $\mathrm{mg} \mathrm{L}^{-1}\left(\mathrm{CaCO}_{3}\right)$ & $*$ & $0 \mathrm{n}=8$ & $0 \mathrm{n}=7$ & $0 \mathrm{n}=8$ & $0 \mathrm{n}=8$ & $0 \mathrm{n}=7$ \\
\hline Cálcio, $\mathrm{mg} \mathrm{L}^{-1}$ & * & $0,03 \pm 0,21 \mathrm{n}=10$ & $0,04 \pm 0,50 \mathrm{n}=9$ & $0,02 \pm 0,27 n=10$ & $0,01 \pm 0,30 \mathrm{n}=10$ & $0,04 \pm 0,26 n=9$ \\
\hline Cloretos, $\mathrm{mg} \mathrm{L}^{-1}\left(\mathrm{Cl}_{2}\right)$ & 250 & $18,3 \pm 5,7 \mathrm{n}=8$ & $19,1 \pm 5,9 \mathrm{n}=7$ & $18,3 \pm 3,5 \mathrm{n}=8$ & $18,8 \pm 4,2 \mathrm{n}=8$ & $20,0 \pm 5,0 \mathrm{n}=7$ \\
\hline Condutividade elétrica, $\mu \mathrm{S} \mathrm{cm}{ }^{-1}$ & $*$ & $487 \pm 49 n=47$ & $542 \pm 50 \mathrm{n}=41$ & $511 \pm 38 n=43$ & $508 \pm 40 n=43$ & $527 \pm 44 n=36$ \\
\hline Dureza total, $\mathrm{mg} \mathrm{L}^{-1}\left(\mathrm{CaCO}_{3}\right)$ & 500 & $2,2 \pm 0,7 \mathrm{n}=10$ & $2,5 \pm 1,8 \mathrm{n}=9$ & $2,2 \pm 1,2 \mathrm{n}=10$ & $2,0 \pm 1,2 \mathrm{n}=10$ & $2,3 \pm 0,9 n=9$ \\
\hline Ferro, $\mathrm{mg} \mathrm{L}^{-1}$ & 0,3 & $<0,3 \mathrm{n}=9$ & $<0,2 \mathrm{n}=9$ & $<0,2 \mathrm{n}=10$ & $<0,2 \mathrm{n}=10$ & $<0,2 \mathrm{n}=8$ \\
\hline Fluoretos, $\mathrm{mg} \mathrm{L}^{-1}$ & 1,5 & $1,39 \pm 1,2 \mathrm{n}=48$ & $0,15 \pm 0,1 \mathrm{n}=42$ & $0,96 \pm 1,1 \mathrm{n}=44$ & $1,44 \pm 1,3 \mathrm{n}=44$ & $1,14 \pm 1,3 \mathrm{n}=38$ \\
\hline Fósforo total, $\mathrm{mg} \mathrm{L}^{-1}(\mathrm{P})$ & $*$ & $0,5 \pm 0,2 \mathrm{n}=10$ & $0,9 \pm 0,2 \mathrm{n}=9$ & $0,8 \pm 0,4 \mathrm{n}=9$ & $0,4 \pm 0,1 \mathrm{n}=10$ & $0,5 \pm 0,2 \mathrm{n}=9$ \\
\hline Magnésio, mg L ${ }^{-1}$ & $*$ & $<0,7 \mathrm{n}=10$ & $<0,8 \mathrm{n}=9$ & $<0,9 n=10$ & $<0,8 \mathrm{n}=10$ & $<0,8 \mathrm{n}=9$ \\
\hline Manganês, mg L ${ }^{-1}$ & $*$ & $<0,03 \mathrm{n}=10$ & $<0,04 \mathrm{n}=9$ & $<0,03 \mathrm{n}=10$ & $<0,04 \mathrm{n}=10$ & $<0,04 \mathrm{n}=9$ \\
\hline $\mathrm{pH}$ & 6,0 a 9,5 & $8,9 \pm 0,2 \mathrm{n}=47$ & $8,83 \pm 0,3 n=41$ & $8,84 \pm 0,3 n=43$ & $8,90 \pm 0,3 n=43$ & $8,81 \pm 0,2 \mathrm{n}=36$ \\
\hline Potássio, mg L ${ }^{-1}$ & $*$ & $<0,3 \mathrm{n}=10$ & $<0,3 n=10$ & $<0,3 n=10$ & $<0,3 \mathrm{n}=10$ & $<0,3 \mathrm{n}=10$ \\
\hline Sódio, $\mathrm{mg} \mathrm{L}^{-1}$ & 200 & $99,5 \pm 13,7 \mathrm{n}=10$ & $107,6 \pm 35,5 n=10$ & $101,1 \pm 22,4 \mathrm{n}=10$ & $95,2 \pm 8,8 n=10$ & $96,2 \pm 9,2 \mathrm{n}=10$ \\
\hline Sólidos totais dissolvidos, $\mathrm{mg} \mathrm{L}^{-1}$ & 1.000 & $353 \pm 36 n=47$ & $393 \pm 37 n=41$ & $370 \pm 27 n=43$ & $368 \pm 29 n=43$ & $382 \pm 32 n=43$ \\
\hline Sulfatos, $\mathrm{mg} \mathrm{L}^{-1}\left(\mathrm{SO}_{4}^{-2}\right)$ & 250 & $25,9 \mathrm{n}=1$ & $23,9 \mathrm{n}=1$ & $26,3 \mathrm{n}=1$ & $25,6 \mathrm{n}=1$ & $26,5 \mathrm{n}=1$ \\
\hline Turbidez, uT & 5 & $<0,3 \mathrm{n}=47$ & $<0,2 \mathrm{n}=41$ & $<0,3 \mathrm{n}=43$ & $<0,3 \mathrm{n}=43$ & $<0,3 \mathrm{n}=36$ \\
\hline
\end{tabular}




\section{CONSIDERAÇÕES FINAIS}

Tendo em vista os aspectos mencionados, os resultados demonstram a eficiência do carvão ativado de osso na adsorção de íons fluoreto - ou seja, a diminuição do excesso de flúor presente na água de abastecimento - e comprovam a sua aplicabilidade em sistemas de abastecimento para uso coletivo, como em escolas, universidade, estabelecimentos comerciais, entre outros. Cabe destacar que, atualmente, os sistemas de desfluoretação continuam sendo monitorados, a fim de confirmar a eficiência do carvão ativado de osso, após a troca da fase estacionária. Contudo, deve-se realizar o monitoramento das variáveis que é de suma importância para potabilidade da água após a instalação do sistema de filtração, para ficarem de acordo com os parâmetros estabelecidos da Portaria n 2914, do Ministério da Saúde.

\section{REFERÊNCIAS}

ABNT - ASSOCIAÇÃO BRASILEIRA DE NORMAS TÉCNICAS. NBR 14908:Aparelho para melhoria da qualidade da água para uso doméstico - Aparelho por pressão. Rio de Janeiro, 2004 a.

ABNT - ASSOCIAÇÃO BRASILEIRA DE NORMAS TÉCNICAS. NBR 15176: Aparelho para melhorias da qualidade da água para uso doméstico - Aparelho por gravidade. Rio de Janeiro, 2004 b.

ANA - Agência Nacional de Águas (Brasil). Cuidando das águas: soluções para melhorar a qualidade dos recursos hídricos. Disponível em:

<http://www.pnuma.org.br/admin/publicacoes/texto/Cuidando_das_aguas_final_baixa.pdf>. Acesso em: 01 mar. 2015.

APHA. American Public Health Association. Standard Methods: for the examination of water \& wastewater. 21 st. ed. Centennial Edition, 2005. 1238 p.

BRASIL. Ministério da Saúde. Portaria n 2914, de 12 de dezembro de 2011. Dispõe sobre os procedimentos de controle e de vigilância da qualidade da água para consumo humano e seu padrão de potabilidade. Diário Oficial União, Brasília, v. 239, p.39-46, 14 dez. 2011, Seção 1.

CATANI, D. B.; HUGO, F. N.; CYPRIANO, S.; SOUSA, M. L. R; CURY, J. A. Relação entre níveis de fluoreto na água de abastecimento público e fluorose dental. Revista de Saúde Pública, São Paulo, v. 41, n. 5, p. 732-739, out. 2007.

COSTA, A. B (Org.). Água \& saúde. 1. ed. Santa Cruz do Sul: EDUNISC, 2010. 
COSTA, A. B.; LOBO, E. A.; SOARES, J; KIRST, A.; Desfluoretação de águas subterrâneas utilizando filtros de carvão ativado de osso. Revista Águas Subterrâneas, São Paulo, n. 3, v. 27, p. 60-70, 2013.

EXTERCKOTER, R. K.; SCHLINDWEIN, S. L. Uso de Indicador no Diagnóstico da Efetividade no Tratamento de Água em uma Estação Pública de Abastecimento. Revista Brasileira de Recursos Hídricos, Porto Alegre, n. 1, v. 13, p. 125-135, jan./ mar. 2008.

FRAZÃO, P.; PERES, M. A.; CURY, J. A. Qualidade da água para consumo humano e concentração de fluoreto. Revista de Saúde Pública, São Paulo, n. 5, v. 45, p. 964-973, out. 2011.

JÚNIOR, A. P. M.; JUNIOR, T. T. N. Perspectivas de efetivação da cobrança pelo uso da água no Brasil com base no caso da porção mineira da bacia do Paraíba do Sul. Geografias Artigos Científicos, Belo Horizonte, n. 1, v. 5, p. 07-21, jan./ jun. 2009.

LOBO, E. A. (Cord.); COSTA, A. B.; KIRST, A.; MACHADO, E. L.; SOARES, J. KUNRATH, I. G.; WETZEL, A. P. Desfluoretação parcial de águas subterrâneas. $6^{\circ}$ Caderno de pesquisa em engenharia de saúde pública. FUNASA, Brasília, p. 75-106, 2013.

MEENAKSHI, R., MAHESHWARI, C. Fluoride in drinking water and its removal. Journal of Hazardous Materials, n. 1, v. 137, p. 456-463, set. 2006.

MOYSÉS, S. J.; MOYSÉS, S. T.; ALLEGRETTI, A. C. V.; ARGENTA, M.; WERNECK, R. Fluorose dental: ficção epidemiológica? Revista Panamericana de Salud Publical Pan American Journal of Public Health, n. 5, v. 12, p. 339-346, 2002.

OMS - Organizacion Mundial de La Salud. Guia para la calidad del agua potable, Genebra, 2 ed., 2005.

RIBEIRO, M. V. Uso de Carvão de Osso Bovino na Defluoretação de Água para Uso em Abastecimento Público. 2011. 75 f. Dissertação (Curso de Pós-Graduação em Engenharia Metalúrgica e de Minas - Mestrado) - Escola de Engenharia da Universidade Federal de Minas Gerais, Belo Horizonte, 2011.

SOARES, J.; COSTA, A. B.; LOBO, E. A.; KIRST, A.; KUNTATH, I. G.; CORD, D. D. Avaliação da qualidade da água tratada por sistemas de filtração utilizando carvão de osso. UNOPAR Científica - Ciências Exatas e Tecnológicas, Londrina, v. 7, p. 05-12, nov. 2008. 